\title{
Author Correction: Ruthenium-catalysed oxidative conversion of ammonia into dinitrogen
}

\author{
Kazunari Nakajima (D), Hiroki Toda (10, Ken Sakata (1) and Yoshiaki Nishibayashi@
}

Correction to: Nature Chemistry https://doi.org/10.1038/s41557-019-0293-y, published online 24 July 2019.

In the version of this Article originally published, the last sentence of the first paragraph incorrectly read: 'However, to the best of our knowledge, catalytic conversion of ammonia into dinitrogen with transition metal complexes has not yet been achieved ${ }^{7}$, although several groups have reported stoichiometric reactivities of transition metal-ammonia complexes with oxidative electron transfer ${ }^{8-10}$ and hydrogen atom abstraction conditions ${ }^{11-13}$.

This sentence has been corrected to read as follows: 'During the review process for this manuscript, Hamann, Smith and co-workers reported the ability of ruthenium polypyridyl complexes to catalyse ammonia oxidation to dinitrogen ${ }^{7}$. Aside from this work, to the best of our knowledge, there are no other reports on the catalytic conversion of ammonia into dinitrogen with transition metal complexes, although several groups have reported stoichiometric reactivities of transition metal-ammonia complexes with oxidative electron transfer ${ }^{8-10}$ and hydrogen atom abstraction conditions ${ }^{11-13}$.

Published online: 26 February 2020

https://doi.org/10.1038/s41557-020-0441-4

๑) The Author(s), under exclusive licence to Springer Nature Limited 2020 